\title{
Technogenic electromagnetic impact zones of electric grid facilities
}

\author{
Fedor Bryukhan ${ }^{1, *}$ and Mariya Amirkhanyan ${ }^{1}$ \\ ${ }^{1}$ Moscow State University of Civil Engineering, Yaroslavskoe sh. 26, 129337 Moscow, Russia
}

\begin{abstract}
Introduction of technogenic physical fields including electromagnetic fields (EMF) to the environment can have an adverse impact on both humans and environment. Development of safety tools protecting against EMF impact involves studying their intensity and assessing EMF spatial extent. This paper proposes a definition of an EMF technogenic impact zone (TIZ) concept based on the instruments lower sensitivity threshold. The paper gives the results of EMF TIZ width assessment based on the measurements of EMF electric and magnetic components intensity generated by power line communication (PLC) along its 200-meter section. The EMF TIZ width was found to be homogeneous along the PLC section. It is noted that the proposed interpretation of EMF TIZ and method of its determination can be generalized for other EMF sources as well, for example, for SHF-radiation sources, tele- and radiotransmission facilities, mobile communication antennas, etc.
\end{abstract}

\section{Introduction}

Intensive use of electric energy resulted in widespread generation of electromagnetic fields $(\mathrm{EMF})$ in residential and industrial developments. One of the most powerful electromagnetic wave sources is the industrial frequency current $50 \mathrm{~Hz}$ (in some countries the $60 \mathrm{~Hz}$ alternating current is used). According to the latest data, the total average annual electricity output in Russia amounts to $215 \mathrm{GW}$. All this electric energy is transported by grid companies operating large-scale electric grid facilities (EGF). According to the latest data, the total length of power line communication (PLC) in Russia exceeds 2.7 million $\mathrm{km}$.

Intensity of electric component of EMF of industrial frequency (EMFIF) emerging in operation of open switchgears, electric power substations, PLC and other EGF increases dramatically as advancing towards the EMFIF source and can reach tens of thousands of $\mathrm{V} / \mathrm{m}$ and higher, exceeding natural background in tens of thousand times [1]. At the same time, there is convincing evidence that even much weaker fields have a harmful effect on humans, fauna, flora and soil $[2,3]$.

Propagation range of the EMFIF generated by EGF elements depends on their voltage class. For overhead PLC it reaches tens and even hundreds of meters. To make EMFIF propagation zone dimensions definite in quantitative terms, there are concepts of EMFIF electric and magnetic components impact zones. Russian standards define electric field

\footnotetext{
*Corresponding author: pniiis-gip@mail.ru
} 
impact zone as a space where electric field intensity at a frequency of $50 \mathrm{~Hz}$ exceeds $5 \mathrm{kV} / \mathrm{m}$, and magnetic field impact zone as a space where electric field intensity exceeds $80 \mathrm{~A} / \mathrm{m}$.

It should be noted that the concept of PLC impact zones as well as of other EGF elements does not provide adequate representation of spatial scales of the territories within which the EMFIF impact is significant. The concept of influence zones of electrical installations is used in the organization of works near electrical installations, as well as in ensuring safety in powerful EMF [4] More justified criterion for the extent of such territories can be technogenic impact zones (TIZ) of certain EGF elements.

According to [5], industrial facility TIZ is defined as the territory around such facility, which is impacted by it resulting in deterioration of air, water, geological environment, etc. However, as emphasized in [6], if we follow this definition and take into account existence of no matter how weak impacts, we can arrive at the obvious conclusion that TIZ is unlimitedly extended. It should obviously be about significant impacts in real conditions too, which can be reliably established in one way or another. The work [6] defines TIZ as the territory around an industrial (economic) facility within which negative changes in the landscape envelope due to facility multifactor impact can be reliably established. This concept of TIZ was developed to assess thermal power plants impact on the environment [6].

The necessity to take into account even very weak levels of EMFIF electric and magnetic components when detecting TIZ is related to the following circumstances.

1. At present, the maximum permissible levels for assessing EMF environmental impact are not generally developed. The only biological object for which the maximum permissible levels are developed and implemented is human.

2. We can assume with a high degree of confidence that exposure to even weak EMFIF has a negative impact on humans and biota. This confidence becomes evident if we consider frequent long stay of living organisms in EMF. In addition, EMF technogenic adversity for environment state appears from pollution definition: "Pollution is everything being in the wrong place, at the wrong time and in the amount alien to nature making its systems unbalanced and differing from usually observed normal condition" [7].

3. Living organisms show sensitivity even to super weak EMF [8]. It is known that EMFIF in addition to humans have a negative impact on animals, especially, on reptiles, birds and insects [1]. For example, insects are noted to increase anxiety and aggression, behavior change. In particular, insects show a tendency to move in the direction of zones with a lower level of EMF strength. Domestic animals grazing near the power line are showing an increased morbidity.

4. Finally, consideration of weak fields when determining TIZ limits is justified from the point of view of EGF environmental hazard presumption.

\section{Planning of researches as part of engineering and environmental surveys}

It seems obvious that TIZ limits can only be established with the help of instrumental monitoring. Electric or magnetic field intensity minimum values, which can reliably be measured, depend on measuring instrument accuracy class. The more accurate the instrument is, the smaller values of EMFIF electrical and magnetic components can be measured and the greater TIZ length can accordingly be detected. Thus, EGF technogenic electromagnetic impact zone can apparently be defined as the territory around the EGF, within which alternating electric and/or magnetic fields generated by such facility can reliably be measured with instruments. The cumulative zone of the two zones established by measuring electric and magnetic fields intensity is set as the most extended. It should be kept in mind that EGF TIZ must be determined at the maximum transmitted electrical power. 
To measure EMFIF as part of engineering survey, the following instruments are used most frequently in Russia:

- VE-meter in $50 \mathrm{~Hz}$ modification - three-component of $50 \mathrm{~Hz}$ industrial frequency electromagnetic field parameters meter;

- EcoMaxima kit - multifunctional set of instruments for alternating EMF intensity measurement;

- P3-81-ECO - combined EMF measuring instrument;

- Octava-110A (E-meter/H-meter).

EGF TIZ is quite simple to identify. To do this, it is sufficient to determine the zone around an EGF, within which an instrument reliably reacts to EMF presence with intensity exceeding the lower threshold of its sensitivity.

\section{Results and discussion}

A section of PLC-110 kV of $200 \mathrm{~m}$ long passing through the territory of the Losinoostrovsky Reserve (Moscow) was taken as a measurement object. A photograph of the investigated territory is shown in Figure 1. This area is relatively flat, covered with forest plantations.

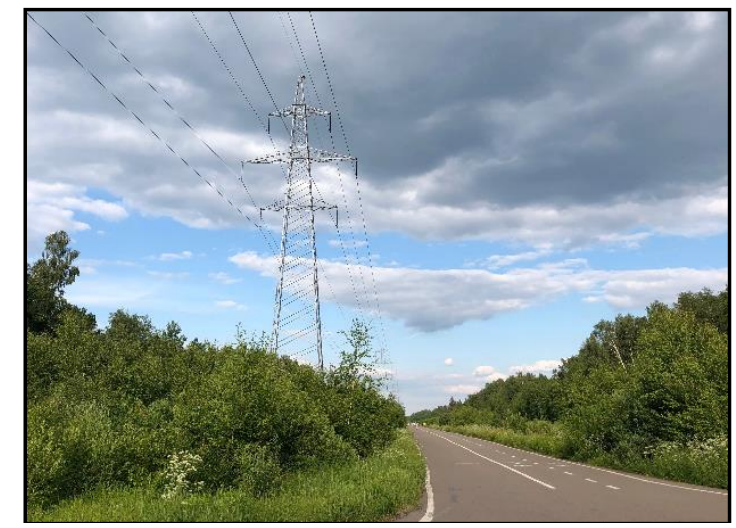

Fig. 1. Photograph of the investigated territory.

Measurements with the use of Octava-110A allowed to determine TIZ dimensions for both electric and magnetic components of EMF. TIZ width for the EMF electric component was approximately $150 \mathrm{~m}$, for the magnetic component - about $60 \mathrm{~m}$ (see Figure 2 and 3). Two conditions were taken as a TIZ limit criterion:

- Option 1. $E>E_{0}, H>H_{0}$.

- Option 2. $E>3 E_{0}, H>3 H_{0}$.

Here, $E_{0}=420 \mathrm{mV} / \mathrm{m}, H_{0}=5 \mathrm{~mA} / \mathrm{m}$, are the sensitivity thresholds for EMF electric and magnetic components, respectively. The second condition of the criterion characterizes other threshold values $3 E_{0}$ and $3 H_{0}$ of the same order as the EMF components sensitivity thresholds. 


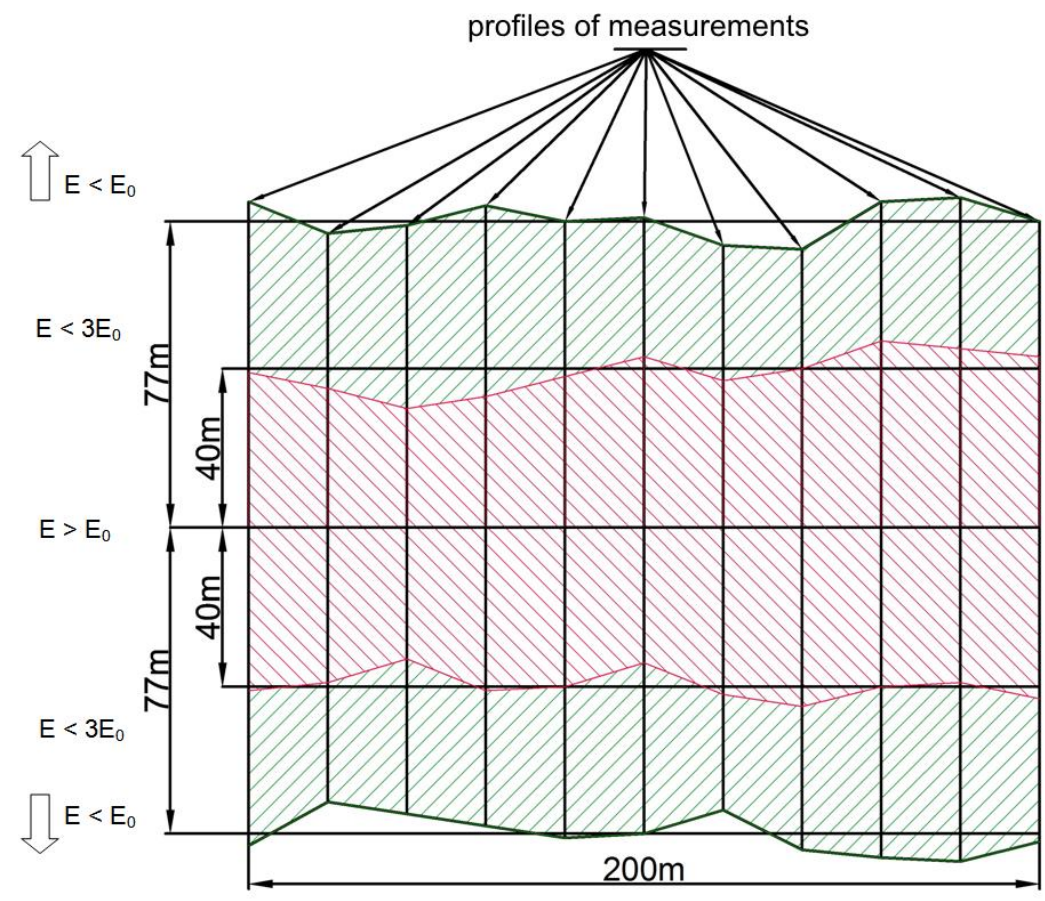

Fig. 2. Limits of EMF electric component technogenic impact zone.

When determining TIZ, the EMF components were measured using 11 profiles across the PLC. Figures 2 and 3 show the relative spatial homogeneity along the PLC section under consideration. For the first criterion condition, electric field TIZ width varies from $140 \mathrm{~m}$ to $166 \mathrm{~m}$, that of magnetic field - from $54 \mathrm{~m}$ to $66 \mathrm{~m}$. Another criterion condition gives an estimate of electric field TIZ width of 66-94 m, that of magnetic field $-30-44 \mathrm{~m}$. Thus, cumulative TIZ width in the first case is estimated as $60 \mathrm{~m}$, and in the other case as $37 \mathrm{~m}$.

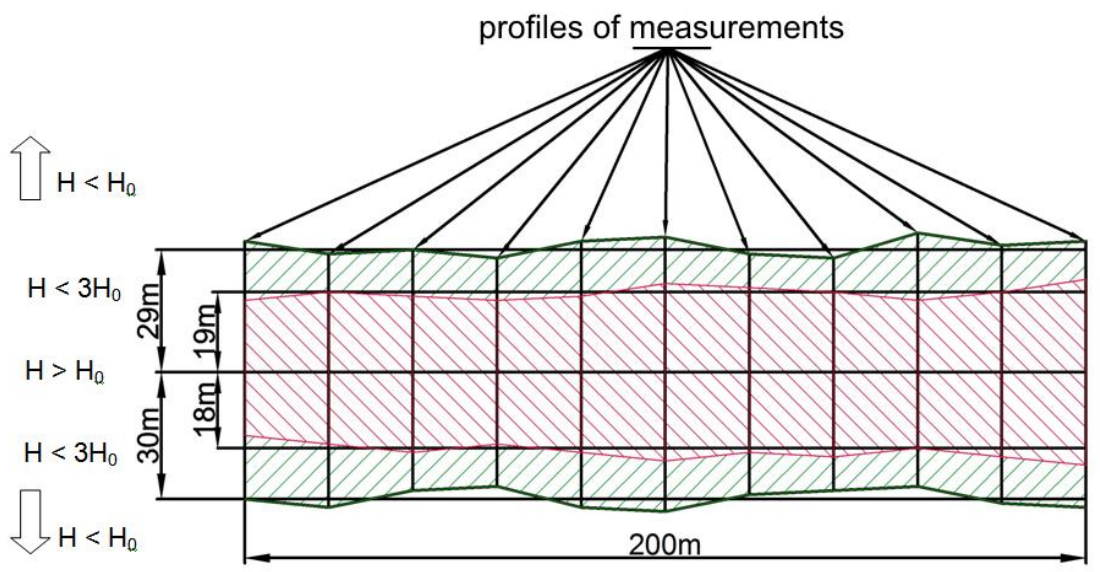

Fig. 3. Limits of EMF magnetic component technogenic impact zone.

According to the Russian sanitary standards on the design of sanitary protection zones (SPZ) for $110 \mathrm{kV}$ PLC, the SPZ is $40 \mathrm{~m}$ wide. The TIZ width determined by the criterion $E$ $>3 E_{0}, H>3 H_{0}$ is closest to such SPZ width. Nevertheless, based on the principle of 
environmental hazard presumption it is more reasonable to use the first criterion condition: $E>E_{0}, H>H_{0}$. Thus, the cumulative TIZ width is estimated as $153 \mathrm{~m}$.

The proposed interpretation of EMF TIZ and method of its determination can be generalized for other EMF sources as well, for example, for SHF-radiation sources, tele- and radio-transmission facilities, mobile communication antennas, etc.

\section{Conclusions}

- The organization of human and environmental protection measures from EMF of industrial frequency provides for the detection of the extent of both the electrical and magnetic components of the EMF.

- Definition of technogenic electromagnetic impact zones of electric grid facilities is proposed and justified.

- Based on the results of field EMF measurements, the EMFIF TIZ limits for overhead PLC-110 kV section are established. TIZ is characterized by spatial homogeneity along the surveyed PLC section. The cumulative TIZ width is estimated as $60 \mathrm{~m}$.

- It is noted that the proposed interpretation of EMF TIZ and method of its determination can be generalized for other EMF sources as well, for example, for SHF-radiation sources, tele- and radio-transmission facilities, mobile communication antennas, etc.

\section{References}

1. Electromagnetic fields and public health: Effects of EMF on the environment (World Health Org., 2005)

2. Environmental, health, and safety guidelines for electric power transmission and distribution (Intern. Finance Corp., Washington, 2007)

3. J.A. Edminister. Schaum's outline of theory and problems of electromagnetics (McGrawHill, NY, 1995)

4. PUE-7. Rules for electrical installations (UVSIZ Publishing House, St. Petersburg, 2005)

5. D.A. Golubeva, N.D. Sorokina (Eds.). Terms and definitions for environmental protection, nature management and environmental safety (SPbGU Publishing House, St. Petersburg, 2001)

6. A.F. Bryukhan, F.F. Bryukhan, A.D. Potapov. Engineering and environmental surveys for construction of thermal power plants (ASV Publishing House, Moscow, 2010)

7. V.V. Snakin. Ecology and environmental protection (Academia, Moscow, 2010)

8. C. Frid, E. Andonegi, J. Depestele et al, Envir. Impact Asses. Rev., 7, (2011) 